\title{
A new approach to selecting coherent pixels for ground-based SAR deformation monitoring
}

\author{
Zheng Wang, Zhenhong Li, Jon Mills \\ School of Engineering, Newcastle University, Newcastle upon Tyne NE1 7RU, UK; \\ z.wang66@newcastle.ac.uk; zhenhong.li@newcastle.ac.uk; jon.mills@newcastle.ac.uk
}

\begin{abstract}
Ground-Based Synthetic Aperture Radar (GBSAR) is a flexible field-based remote sensing technology that, together with interferometric SAR (InSAR), has proven to be a powerful and effective tool for deformation monitoring. The Small Baseline Subset (SBAS) algorithm represents a typical advanced InSAR technique that extracts distributed scatterers from a network of interferograms for the measurement of time series displacement. However, it is well known that coherent points are variable from one interferogram to another, which renders time series analysis complicated. This study therefore proposes an effective approach to selecting coherent pixels from a network of interferograms, aiming to maximize the density of selected pixels and optimize the reliability of GBSAR time series analysis. A pixel is selected for the entire analysis if its coherent phase is capable of forming a full-rank coefficient matrix in the network inversion. A full-rank matrix means the pixel-dependent subset network is connected. Combining with the accurate estimation of coherence and interferometric phase based on sibling pixels identified from non-local windows, the proposed approach enables the selection of not only qualified partially coherent pixels but also persistent scatterers. The proposed approach has been incorporated into a bespoke GBSAR time series analysis chain for deformation monitoring, from which a mean velocity map, displacement time series and atmospheric phase delays can be determined. To validate the approach, experiments on two GBSAR datasets were performed. In both studies, sufficient coherent pixels were selected, suggesting the feasibility of the proposed coherent pixel selection algorithm. Displacement time series at the level of a few sub-millimeters were observed for both datasets, indicating the feasibility of the newly-developed GBSAR time series analysis chain for deformation monitoring, which is believed to lead to a wide range of scientific and practical applications.
\end{abstract}

Keywords: Ground-Based Synthetic Aperture Radar (GBSAR), interferometry, time series analysis, deformation monitoring, partially coherent pixels, Small Baseline Subset (SBAS) algorithm

\section{Introduction}

Hazards involving ground movements such as landslides, mudflows and the collapse of infrastructures are regular occurrences globally, often leading to significant human and economic losses. Such scenarios require effective monitoring of ground movements, which can give insight into mechanisms and triggering factors of hazardous events or act as the basis for mitigating risk, e.g. understanding maintenance and remedial measures, early warning and rapid decision-making for countermeasures or evacuation. Ground-Based Synthetic Aperture Radar (GBSAR) is a field-based imaging system offering users enhanced capabilities in monitoring surface displacements [1-3]. More recently, GBSAR has proven to be a powerful remote sensing tool for deformation monitoring applications [3-5]. In comparison to spaceborne platforms, GBSAR has inherent advantages in terms of flexibility and portability which usually leads to a stack of consecutive acquisitions and offers opportunities for time series analysis. Interferometric Synthetic Aperture Radar (InSAR) time series analysis is the advanced form of the differential InSAR technique to identify and quantify ground movements based on multiple interferograms generated from a stack of SAR images [6-8].

Usually, a critical step in the processing of any InSAR time series analysis is the selection of coherent 
pixels with high Signal-to-Noise Ratio (SNR) in interferometric phase [9]. Further analysis and interpretation is then conducted only on the selected pixels. Amplitude Dispersion Index (ADI) and coherence are two commonly used criteria [10] for the selection of coherent pixels as they are strongly correlated with the standard deviation of the interferometric phase noise $[6,11]$. According to the selection strategy and the processing of selected pixels, a number of InSAR time series analysis algorithms have been developed in the last two decades [12]. These algorithms fall into two broad categories: (a) Persistent Scatterer (PS) InSAR [6] which targets pixels with consistent scattering properties in time and viewing geometry, making this technique more suitable for artificial surfaces with sufficient back scatterers; (b) the more general Small Baseline Subset (SBAS) algorithm which uses distributed scatterers and singular value decomposition to connect independent unwrapped interferograms in time [13,14].

PS techniques are commonly based on a single-master configuration, with the main drawback of such techniques being the low spatial density of targets that behave coherently over the whole observation span [15]. By contrast, SBAS approaches construct a network of interferograms with multiple master images and small baselines [16]. However, as pointed out in [17], coherent points are variable from one interferogram to another, rendering time series analysis complicated. In other words, there are some partially coherent pixels (PCPs) that are coherent in some interferograms but not in others. Regarding the selection of coherent pixels in a redundant network, Crosetto, et al. [18] selected only pixels for which coherence was greater than a given threshold for all interferograms. PCPs were discarded in this approach, meaning the loss of some useful observations. Perissin and Wang [15] formed a pixel-dependent network for each pixel by imposing a threshold on the coherence and only pixels with a connected network were analyzed. The subset of interferograms with respect to the minimum spanning tree graph in the network were used for the estimation of height and deformation trends. Such an approach fails to make the most effective use of redundancies and thus degrades the accuracy since a higher redundancy implies a more reliable displacement rate estimation. Therefore, a new selection criterion of PCPs is proposed in this paper that aims to maximize the density of selected pixels and optimize the reliability of GBSAR time series analysis by making the most of coherent phase redundancies.

Specifically, the method proposed in this paper forms a redundant interferogram network with a specified baseline threshold. A pixel-dependent matrix is then constructed for each pixel based on its coherence occurrences over all interferograms in the network. Pixels with a full-rank matrix are selected for further time series analysis. The proposed criterion enables the selection of not only qualified PCPs, but also persistent scatterers that behave coherently over all interferograms. Interferometric phase observations of selected pixels are spatially filtered and unwrapped. The inversion of the deformation trend is achieved only based on the coherent interferometric phase after filtering and unwrapping, which guarantees a reliable solution. In principle, the proposed approach supports any co-registered SAR datasets, but this paper only focuses on GBSAR deformation monitoring. The methodology is described in Section 2. The feasibility of the proposed method is verified by experimentation with two real-world GBSAR datasets in Section 3. The proposed approach is compared with the selection of coherent pixels using a single-pair GBSAR images and PS selection based on ADI in Section 4, where the justification of related parameters adopted in the proposed method are also discussed. Conclusions are drawn in Section 5.

\section{Methodology}

\subsection{Selection criterion of coherent pixels}

The phase difference at a point between two SAR images is called interferometric phase [19], which is the superposition of many terms including the topographic component, change resulted from surface movement in the light-of-sight (LOS) direction, variation of atmospheric delays, ambiguous cycles and noise. Analysis starts with a stack of SAR single-look-complex (SLC) images $\left(E_{0}, E_{1}, \ldots, E_{N}\right)$ relative to the same 
illuminated region, acquired at times $\mathbf{t}\left(t_{0}, t_{1}, \ldots, t_{N}\right)$ in the chronological order. A redundant network of $L$ interferograms formed by SLC images is assumed. The differential interferometric phase for a target between the SAR acquisitions at times $t_{M}$ (for the master image) and $t_{S}$ (for the slave image), can be written as:

$$
\varphi_{t_{M} t_{S}}^{w}=\varphi_{t_{M} t_{S}}^{\text {Topo }}+\varphi_{t_{M} t_{S}}^{\text {disp }}+\varphi_{t_{M} t_{S}}^{a t m}+\varphi_{t_{M} t_{S}}^{\text {noise }}-2 n \pi
$$

where the superscript symbol $w$ of the interferometric phase $\varphi_{t_{M} t_{S}}^{w}$ denotes that the value is wrapped into the range $[-\pi, \pi]$. The interferometric phase is actually a relative value due to the integer ambiguity $n$ [12]. To obtain the absolute value, the recovery of ambiguity is required through a process known as phase unwrapping [20]. Thereafter, the unwrapped phase can be written as:

$$
\varphi_{t_{M} t_{S}}=\varphi_{t_{M} t_{S}}^{\text {Topo }}+\varphi_{t_{M} t_{S}}^{\text {disp }}+\varphi_{t_{M} t_{S}}^{a t m}+\varphi_{t_{M} t_{S}}^{\text {noise }}
$$

where the topographic term $\varphi_{t_{M} t_{S}}^{T o p o}$ is a function of the perpendicular spatial baseline [19]. Unlike spaceborne SAR, GBSAR data can be acquired both continuously and discontinuously [21]. Continuous operation offers a zero-baseline geometry, thus the topographic phase component is always zero. In a discontinuous campaign, topographic contributions arising from small repositioning errors can be corrected by treating it as a spatially smooth signal $[3,22]$. Otherwise, the topographic term can be removed through the provision of a digital terrain model of the monitoring area and the precise geometry configuration of the radar equipment in the event of a significant spatial baseline. Without loss of generality, there are always at least three other terms that play a role in GBSAR interferometry [2]:

$$
\varphi_{t_{M} t_{S}}=\varphi_{t_{M} t_{S}}^{\text {disp }}+\varphi_{t_{M} t_{S}}^{a t m}+\varphi_{t_{M} t_{S}}^{\text {noise }}
$$

The goal of InSAR time series analysis for deformation monitoring is to obtain the deformation time series, denoted as $d_{t_{i}}^{\text {disp }}(i=1, \cdots, N)$ with respect to a reference acquisition $t_{0}$. As recognised in previous studies $[13,23]$, the mean velocity between time-adjacent acquisitions is a preferable choice in InSAR time series analysis in order to avoid large discontinuities in cumulative deformations and to obtain a physically reliable solution. In this case, no prior knowledge about the deformation is required in the network inversion. The displacement term in the interferometric phase can be expressed as:

$$
\varphi_{t_{M} t_{S}}^{\text {disp }}=\frac{4 \pi}{\lambda}\left[d_{t_{M}}^{\text {disp }}-d_{t_{S}}^{\text {disp }}\right]=\frac{4 \pi}{\lambda} \sum_{k=M}^{S-1} v_{k}^{\text {disp }} \Delta t_{k}=\sum_{k=M}^{S-1} \varphi_{t_{k} t_{k+1}}^{\text {disp }}
$$

where $v_{k}^{\text {disp }}$ is the displacement velocity and $\varphi_{t_{k} t_{k+1}}^{\text {disp }}$ is the associated phase change between the $k^{\text {th }}$ and the $(k+1)^{t h}$ acquisitions and $t_{k}$ is the time interval between them. Similarly, the time-series atmospheric variation is denoted as $d_{t_{i}}^{a t m}(i=1, \cdots, N)$ and the atmospheric phase contribution in the interferometric phase can be written as:

$$
\varphi_{t_{M} t_{S}}^{a t m}=\frac{4 \pi}{\lambda}\left[d_{t_{M}}^{a t m}-d_{t_{S}}^{a t m}\right]=\sum_{k=M}^{S-1} \varphi_{t_{k} t_{k+1}}^{a t m},
$$

where $\varphi_{t_{k} t_{k+1}}^{a t m}$ represents the atmospheric phase variation between the $k^{\text {th }}$ and the $(k+1)^{t h}$ acquisitions. Together with Equations (4) and (5), the matrix form with respect to Equation (3) can be generalized for the entire interferogram network:

$$
\left\{\begin{array}{l}
\mathbf{B}_{L \times N} \boldsymbol{\Phi}_{N \times 1}=\boldsymbol{\delta} \boldsymbol{\Phi}_{L \times 1}+\boldsymbol{\varepsilon}_{L \times 1} \\
\boldsymbol{\Phi}=\left[\begin{array}{llll}
\left(\varphi_{t_{0} t_{1}}^{d i s p}+\varphi_{t_{0} t_{1}}^{a t m}\right. & \left(\varphi_{t_{1} t_{2}}^{d i s p}+\varphi_{t_{1} t_{2}}^{a t m}\right) & \ldots & \left(\varphi_{t_{N-1} t_{N}}^{d i s p}+\varphi_{t_{N-1} t_{N}}^{a t m}\right.
\end{array}\right]^{\mathrm{T}} \\
\boldsymbol{\delta} \boldsymbol{\Phi}=\left[\begin{array}{lllll}
\varphi_{t_{1} t_{2}} & \ldots & \varphi_{t_{M} t_{S}} & \ldots & \varphi_{t_{N-1} t_{N}}
\end{array}\right]^{\mathrm{T}}
\end{array}\right.
$$


where $\mathbf{B}$ is the coefficient matrix; $\boldsymbol{\Phi}$ is the matrix containing the incremental time series of phase change with respect to the superposition of both displacement and atmospheric variation; $\boldsymbol{\delta} \boldsymbol{\Phi}$ is the matrix of redundant unwrapped interferometric phase $\varphi_{t_{M} t_{S}} ; \boldsymbol{\varepsilon}$ is the noise matrix. Base on Equation (6), the inversion can be performed via least squares using reliable interferometric phase with high SNR and low noise for a reliable solution. Thereafter, to obtain the displacement trend, the atmospheric variations should be compensated by any suitable methods [23, 24].

Within this context, a new approach for the selection of proper coherent pixels is proposed. Specifically, a threshold is imposed on coherence to determine whether a pixel is coherent for one interferogram or not. The coherence of the pixel above the threshold means an acceptable phase quality of the corresponding interferometric phase in the inversion. Considering a number of pixels are partially coherent in the network, the coherence occurrences with respect to a pixel must ensure Equation (6) is a well-determined system, namely the rank of matrix $\mathbf{B}$ is equal to the number of unknowns. Accordingly, all elements in the $k^{\text {th }}$ row of $\mathbf{B}$ will be set as zero if the coherence on the $k^{\text {th }}$ interferogram is lower than the specified threshold. The selection criterion is that provided the matrix $\mathbf{B}$ related to a particular pixel is full-rank, then this pixel is selected for the time series analysis. Consequently, the constructed matrix $\mathbf{B}$ is based on the coherence occurrences over the entire interferogram network. For instance, it is assumed that there is a vector of interferograms $\left(I_{0,1}, I_{0,2}, I_{1,2}, I_{1,3}, I_{2,3}\right)$ formed by a vector of images $\left(E_{0}, E_{1}, E_{2}, E_{3}\right)$ acquired at times $\left(t_{0}, t_{1}, t_{2}, t_{3}\right)$ with $t_{0}$ as the reference. For a pixel that is only coherent on $I_{0,1}, I_{1,3}$ and $I_{2,3}$, the corresponding matrix $\mathbf{B}$ is:

$$
\mathbf{B}=\left[\begin{array}{lll}
1 & 0 & 0 \\
0 & 0 & 0 \\
0 & 0 & 0 \\
0 & 1 & 1 \\
0 & 0 & 1
\end{array}\right] .
$$

As $\mathbf{B}$ is a full-rank matrix in this case, this pixel is selected. A completely coherent pixel always has a full-rank matrix $\mathbf{B}$, thus can be selected by the proposed criterion. A pixel, associated with a full-rank $\mathbf{B}$, is selected for time series analysis. A full-rank $\mathbf{B}$ means that the interferogram network is connected and the inversion in the time series estimation via least squares is enabled using only the coherent phase of this pixel. The proposed method makes the most of redundant observations and allows an adjustment to be made in order to obtain a final reliable value for the unknown.

\subsection{Estimation of coherence and interferometric phase}

The coherence for a pixel between two SAR images is estimated based on $K$ neighboring pixels [25]:

$$
\hat{\gamma}=\frac{\sum_{i=1}^{K} s_{1 i} s_{2 i}^{*}}{\sqrt{\sum_{i=1}^{K}\left|s_{1 i}\right|^{2}} \sqrt{\sum_{i=1}^{K}\left|s_{2 i}\right|^{2}}}=\hat{\rho} \cdot e^{j \widehat{\varphi}},
$$

where $s_{1 i}$ and $s_{2 i}$ are the complex signal values of the SAR image pair for the $i^{\text {th }}$ sample. $\hat{\rho}$ is the maximum likelihood coherence magnitude and $\hat{\varphi}$ is the estimated interferometric phase. Usually, $K$ pixels are selected from a local rectangular window around the pixel of interest [17]. However, $K$ pixels in the local "boxcar" window are likely to indiscriminately average neighboring pixels, leading to overestimations of the coherence and resolution losses due to the local heterogeneity $[17,26]$.

To overcome these limitations, the proposed method uses a similarity measure to identify resembling pixels from a larger non-local window. Based on a stack of N SLC images, the similarity measure between two pixels at $\left(\mathrm{x}_{1}, r_{1}\right)$ and $\left(\mathrm{x}_{2}, r_{2}\right)$ is defined as [27]:

$$
S=1-\left|\bar{A}\left(\mathrm{x}_{1}, r_{1}\right)-\bar{A}\left(\mathrm{x}_{2}, r_{2}\right)\right| /\left(\bar{A}\left(\mathrm{x}_{1}, r_{1}\right)+\bar{A}\left(\mathrm{x}_{2}, r_{2}\right)\right),
$$

where the symbol $|\cdot|$ represents the absolute value operation; $\bar{A}\left(\mathrm{x}_{i}, r_{i}\right)(i=1,2)$ is the mean amplitude of a pixel at $\left(\mathrm{x}_{i}, r_{i}\right)(i=1,2)$ over the $N$ SLC images. The similarity is based on the mean amplitude and the similarity between two pixels with identical mean amplitude is one. The similarity ranges from zero to one, 
with a larger value implying greater resemblance of the pixel pair. A candidate is considered as a sibling of the current pixel when the similarity between them is greater than a specified threshold, for which typical values are 0.85-0.95 and the explanation for which is given in [27]. In this study, the siblings identified by the similarity measure are used as $K$ pixels for the estimation of coherence and the siblings-based coherence magnitude $\hat{\rho}$ is further estimated based on the second kind statistics [28, 29]:

$$
\overline{\hat{\rho}}=\exp \left(\frac{1}{K} \sum_{l=1}^{K} \ln \left(\hat{\rho}_{l}\right)\right) \text {. }
$$

The coherence magnitude obtained by second kind statistics is less biased and the variance of the log-estimate is globally lower than that of the regular estimate [28, 29]. In addition, filtering is firstly applied to improve the SNR of the interferometric phase of the selected pixels. The filtered interferometric phase $\hat{\varphi}$ for each pixel is achieved by the non-local averaging of the complex values of its siblings:

$$
\left\{\begin{array}{rl}
e^{i \widehat{\varphi}} & =\sum_{l=1}^{K} e^{i w_{l} \varphi_{l}} \\
w_{l} & =\overline{\hat{\rho}}_{l} / \sum_{l=1}^{K} \overline{\hat{\rho}}_{l}
\end{array} .\right.
$$

The unwrapped phase of detected pixels is subsequently obtained using the Minimum Cost Flow approach [30].

\subsection{Compensation of atmospheric variations}

The inversion can be implemented via least squares using filtered and unwrapped phase for each selected coherent pixel. The inverted result at this stage is the sum of the surface displacements and the atmospheric variations. To obtain precise displacement time series, any atmospheric artefacts must be properly compensated, which can be approached by means of different techniques [24]. The proposed approach adopts two previous representative works $[31,32]$ to achieve this. For scenarios with smooth topography, the atmospheric variations are modelled as a linear function of range under the medium homogeneity hypothesis [32] and separated from the displacements. In scenes with steep topographic variations, the assumption of spatial homogeneity no longer applies due to the fluctuations of atmospheric parameters such as temperature, pressure, and humidity on the spatial domain [31]. Therefore, for cases with steep topography, atmospheric variation can be considered as a range- and height-dependent model and compensated with the support of the height information [31].

\section{Experimental results}

\subsection{Overview of the datasets}

A MetaSensing's FastGBSAR system [33] was used for data collection. The sensor system operates at $\mathrm{Ku}$ band with a wavelength $(\lambda)$ of $17.4 \mathrm{~mm}$. The repeat interval (i.e. temporal resolution) of data acquisition is adjustable and the fastest acquisition speed with full spatial resolution is $10 \mathrm{~s}$. Two datasets were collected to verify the proposed approach.

The first dataset (Dataset I) was acquired by mounting the radar system on a stationary concrete plinth, continuously observing a cliff on the north side of Tynemouth Priory and Castle, Tynemouth, UK. The monitoring site mainly comprises a cliff face, the castle buildings, some areas of sandy beach and the sea on the south side of King Edward's Bay. More information about the data collection is illustrated in Figure 1. In Dataset I, there were 30 SLC images of VV polarization with a temporal resolution of four minutes. The whole period of acquisition time was two hours. The dimension of each image was 294 by 254 pixels, with spatial resolution was $0.75 \mathrm{~m}$ in the range direction and five milliradians in the azimuth. 


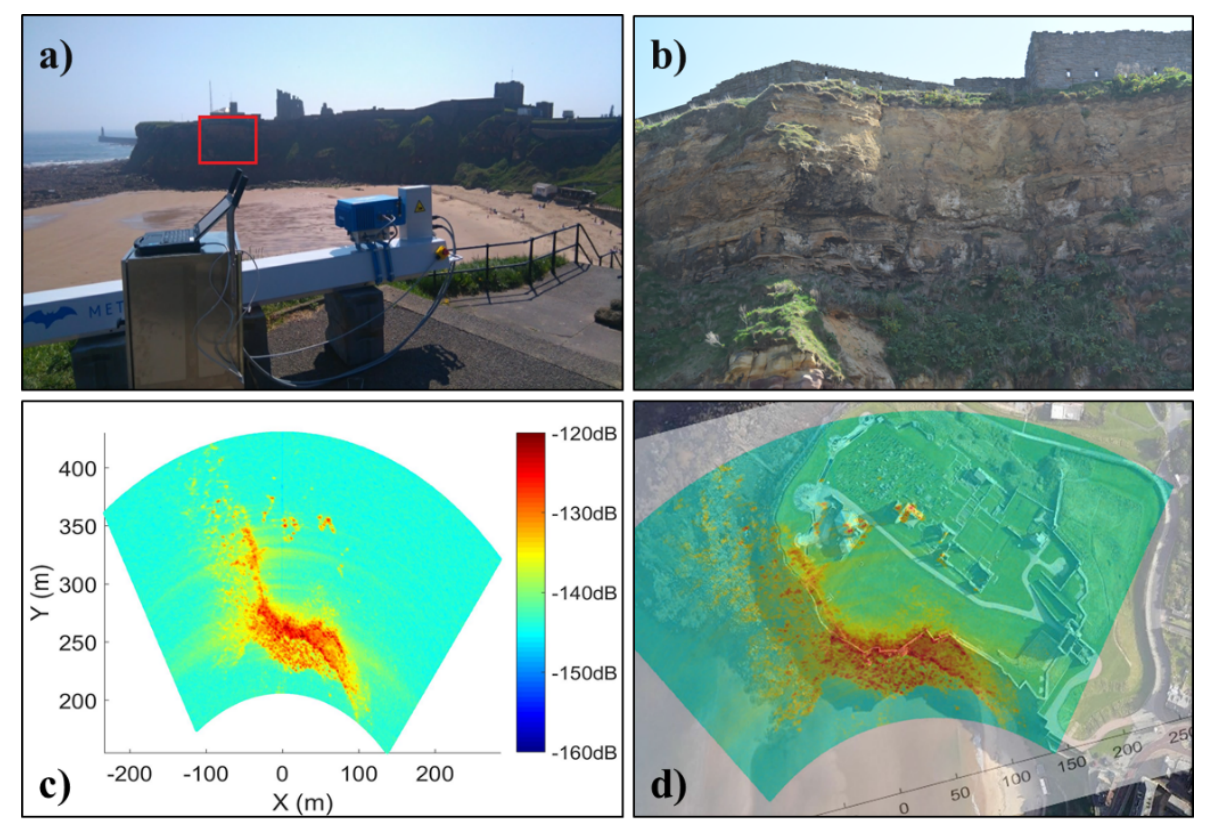

Figure 1. An overview of Dataset I. (a) The deployment of the FastGBSAR system for data collection. (b) A close-up of the cliff face with respect to the area marked in the red box in (a), which mainly consists of outcrops, bare earth and vegetation, and a section of a man-made stone wall. (c) The mean amplitude image of all GBSAR SLCs in Dataset I. (d) Approximate alignment and co-registration of the mean amplitude image with the planimetric view of the observing site in Google Earth.

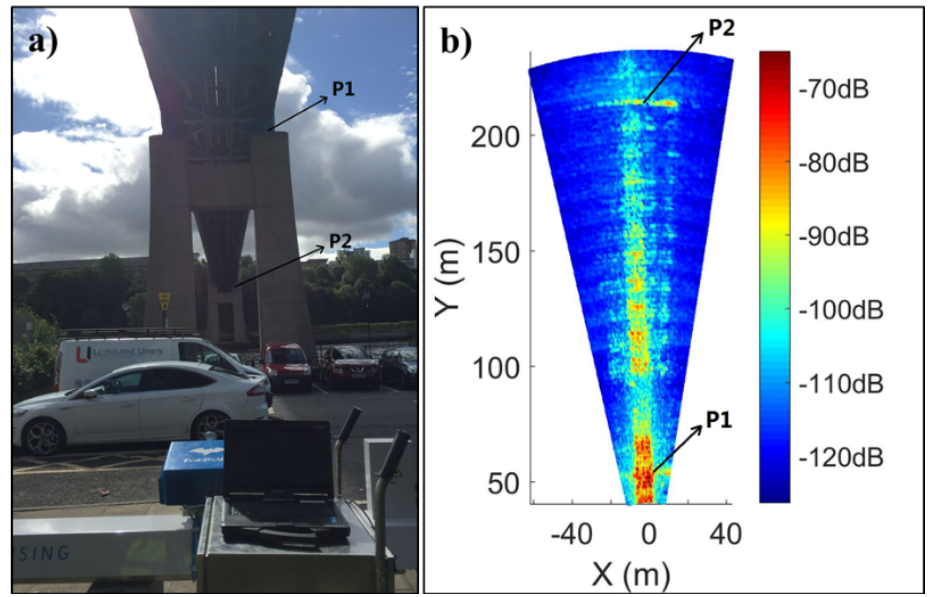

Figure 2. An overview of Dataset II. (a) The deployment of the FastGBSAR system for data collection. (b) The mean amplitude image of this dataset, shown in decibel. Two reference points are identified, based on the relative geometry parameters between the bridge and the radar system, added to assist interpretation of the GBSAR image geometry.

The second dataset (Dataset II) was collected by monitoring the Queen Elizabeth II Metro Bridge, spanning the River Tyne in Newcastle upon Tyne, UK. The FastGBSAR unit was tilted up to an angle of 30 degrees to scan the bridge superstructure which has a steel truss with fabricated box chords construction. The four concrete piers of the bridge and their expansion joints were also imaged. An overview of the site is given in Figure 2. Dataset II consisted of 16 acquisitions collected within two and a half minutes with an equal repeat interval of 10 seconds. The dimension of each image was 256 by 88 pixels. The polarization and spatial resolution of this dataset were identical to the first dataset. A train crossed the bridge during the period of data acquisition, taking around 16 seconds from entering the bridge to completely exiting. Consecutive acquisitions over this short period of time were used to detect the movements of the bridge as the train traveled cross it. 


\subsection{GBSAR time series analysis}

The temporal baseline constraint was set as 24 minutes for Dataset I, considering the computational efficiency and the temporal decorrelation, the justification for which is given in Section 4. Thus, one image was used to generate interferograms with its six previous and six subsequent images and there were 159 interferograms in the network. A full combination of interferograms can provide the most redundant observations, but it also increases the computation load and degrades the real-time performance in time-critical applications. The coherence threshold value was set as 0.45 and other parameters related to non-local coherence estimation and phase filtering were set as follows: the similarity threshold was empirically set as 0.85 ; the non-local window size was 15 by 15 ; a minimum of 10 siblings were kept for each pixel for a reliable estimation.

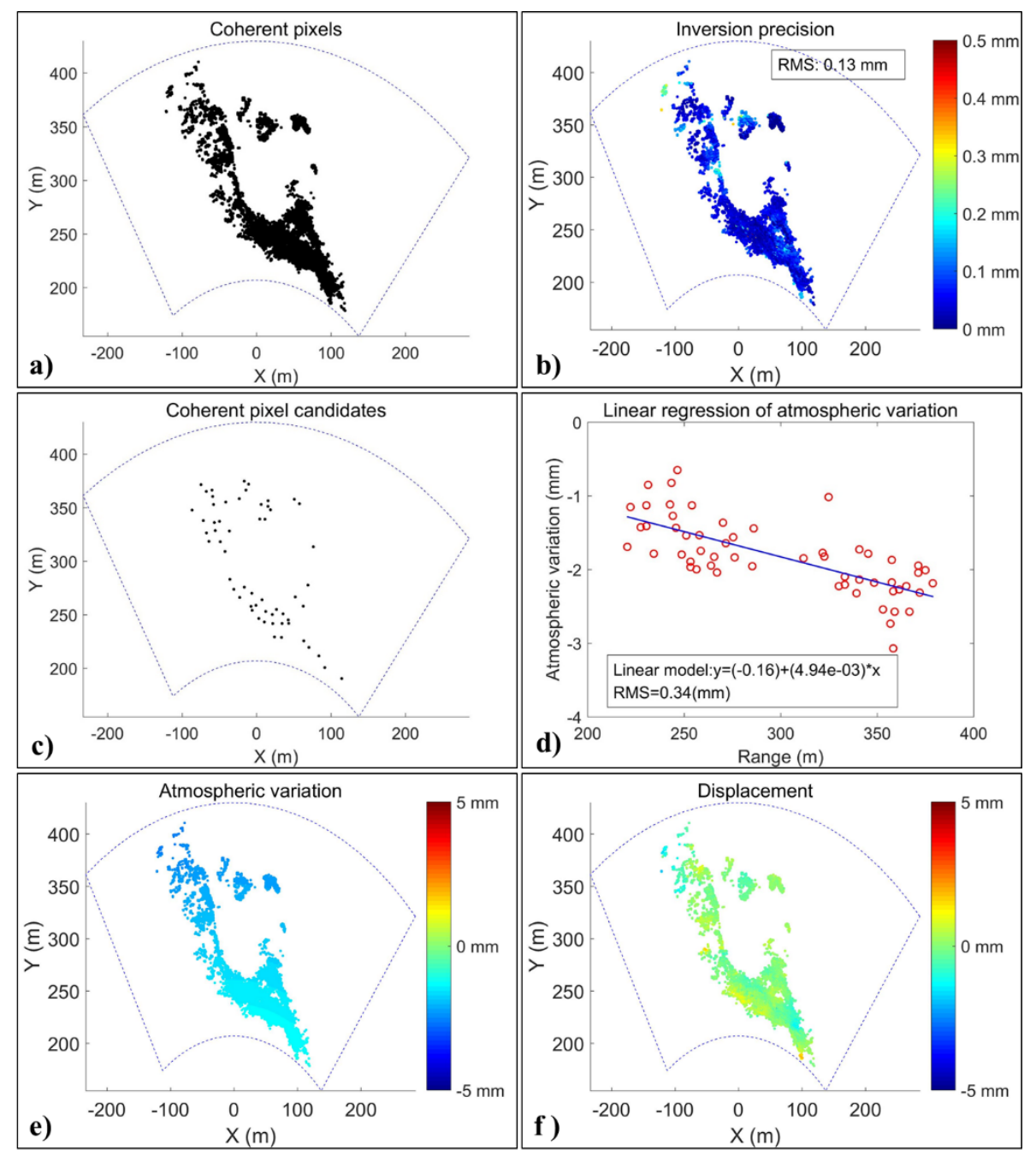

Figure 3. Results of the GBSAR time series analysis. (a) Coherent pixels. (b) Inversion precision (Appendix A). (c) Coherent pixel candidates. (d) Linear regression of atmospheric variations. (e) The atmospheric variation map over the entire period of acquisition (two hours). (f) The cumulative displacement map over the whole period of acquisition time (two hours).

The results of Dataset I are summarized in Figure 3. The number of detected coherent pixels was 5,068 (Figure 3(a)), consisting of 2,042 fully- and 3,026 partially-coherent pixels. Further analysis was carried out only on detected coherent pixels. Least squares inversion with redundant observations allows a precision assessment of the solution. The inversion precision for each pixel was achieved on the basis of phase residuals, with the definition of inversion precision for each pixel given in Appendix A. The overall precision is appraised 
by the root mean square (RMS) of the inversion precision values. Small precision values were achieved in this experiment, as summarized in Figure 3(b), implying a high consistency of the filtered and unwrapped interferometric phase in the least squares inversion. Drawing on previous studies [32,34], a subset of highcoherent pixels, termed coherent pixel candidates (Figure 3(c)), were selected by spatial gridding whereby each candidate had the best coherence within a grid unit. Coherent pixel candidates were used to perform the linear regression of atmospheric variations with the assumption that their displacement components were randomly distributed along the range. The regression result is summarized in Figure 3(d), which shows a reasonable trend that atmospheric variation increases with the range. Residuals of the linear regression can be mainly attributed to atmospheric turbulence and stratification [31, 35]. Based on the linear model in Figure 3(d), the atmospheric variations of all coherent pixels were compensated, and thus separated from any cumulative displacements. The modelled atmospheric variation map over the whole acquisition period (two hours) is shown in Figure 3(e) and the final cumulative displacement map is shown Figure 3(f). It is clear that the atmospheric variation dominates the change and no significant local signals can be found in the final deformation map. Moreover, the cumulative displacements of all coherent pixels are within $2 \mathrm{~mm}$, which can be contributed by observation noise and the residuals of atmospheric compensation. Thus, it is a fair conclusion to state the cliff and castle buildings were stable over the observation time.

Regarding Dataset II and the bridge monitoring, deformation took place in a short period when a train travelled across the bridge. To produce a quick response for the fast-changing process, the temporal baseline constraint was set as half a minute for Dataset II. In this case, each image was involved in the interferogram generation with its three previous and three subsequent images. Thus, 42 interferograms were formed in the redundant network. Based on the priori geometry information about the bridge and the radar system, a shadow zone, caused by the near expansion joint and ranging from $70 \mathrm{~m}$ to $90 \mathrm{~m}$, was masked out before the selection of coherent pixels. The coherence threshold was set as 0.6 to select qualified coherent pixels. The parameters related to the non-local estimation were the same as the first dataset. In this experiment, a total of 5,720 pixels were selected, comparing 4,446 fully- and 1,274 partially-coherent pixels (Figure 4(a)). The RMS of the inversion precision was $0.08 \mathrm{~mm}$ (Figure 4(b)).

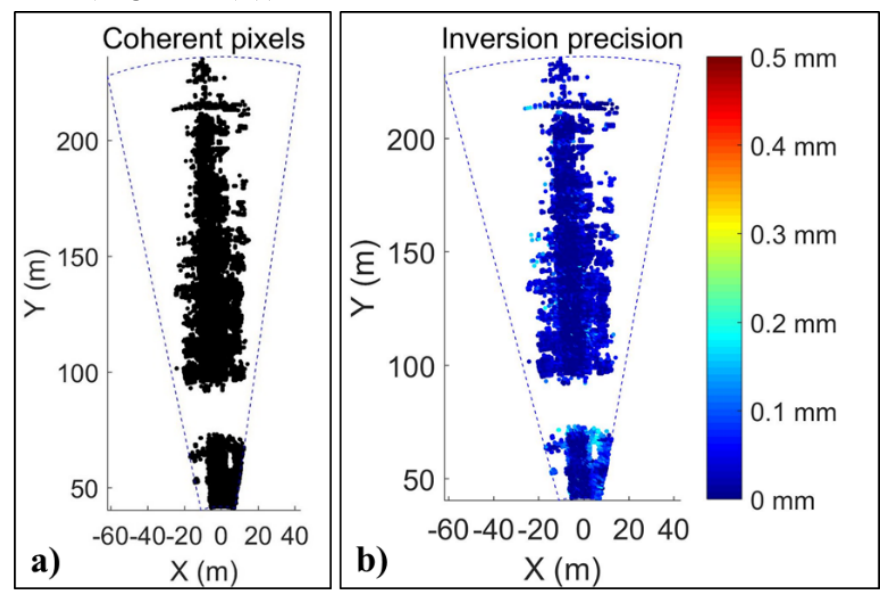

Figure 4. Results of Dataset II. (a) Detection of coherent pixels. (b) Inversion precision of coherent pixels.

As acquisition time for this dataset was only two and a half minutes, an assumption made was that the environmental conditions were stable over this extremely short observation period. In other words, the atmospheric phase change was deemed to be zero and not taken into account in time series analysis. Based on this assumption, the time series of displacement was achieved without the compensation for the atmospheric artefacts. To highlight the bridge deformation as the train travelled across it, the incremental displacement maps with regard to coherent pixels are shown in Figure 5. 


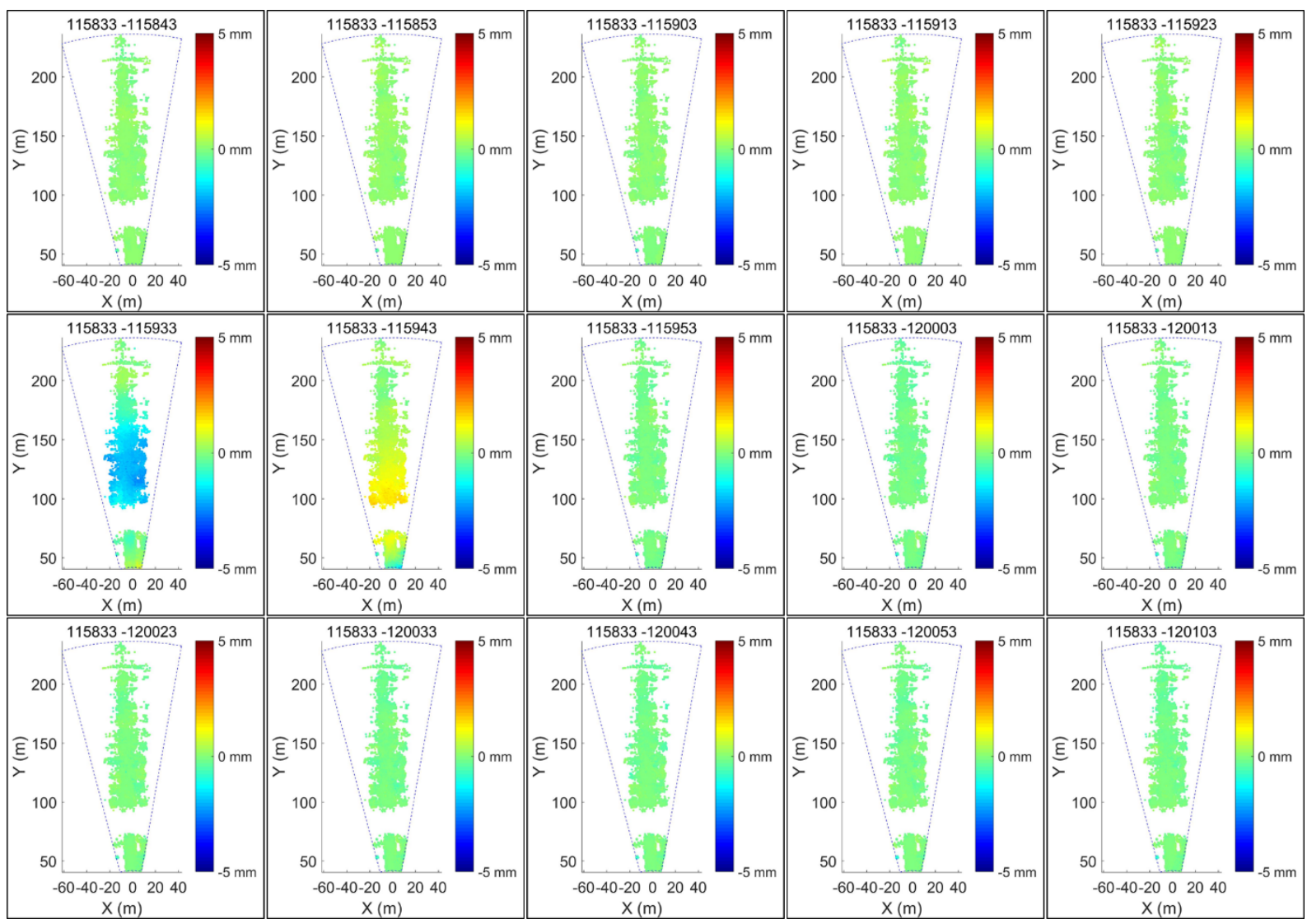

Figure 5. Time series of cumulative displacements at selected coherent pixels. The first image acquired at 11:58:33 was selected as the reference. A train crossed the bridge, resulting in significant deformation signals in the $6^{\text {th }}$ and $7^{\text {th }}$ displacement maps (first two of the second row).

A synchronous video shows that a train crossed the bridge from the far side of the bridge (relative to the location of the FastGBSAR) at around 11:59:28 and completely exited the nearest end at around 11:59:44, taking approximately 16 seconds and spanning two FastGBSAR acquisitions created at UTC times 11:59:33 and 11:59:43, respectively. Constrained by the field of view of the video camera, the complete crossing of the bridge could not be recorded, three video frames are given in Figure 6 to illustrate the situation.

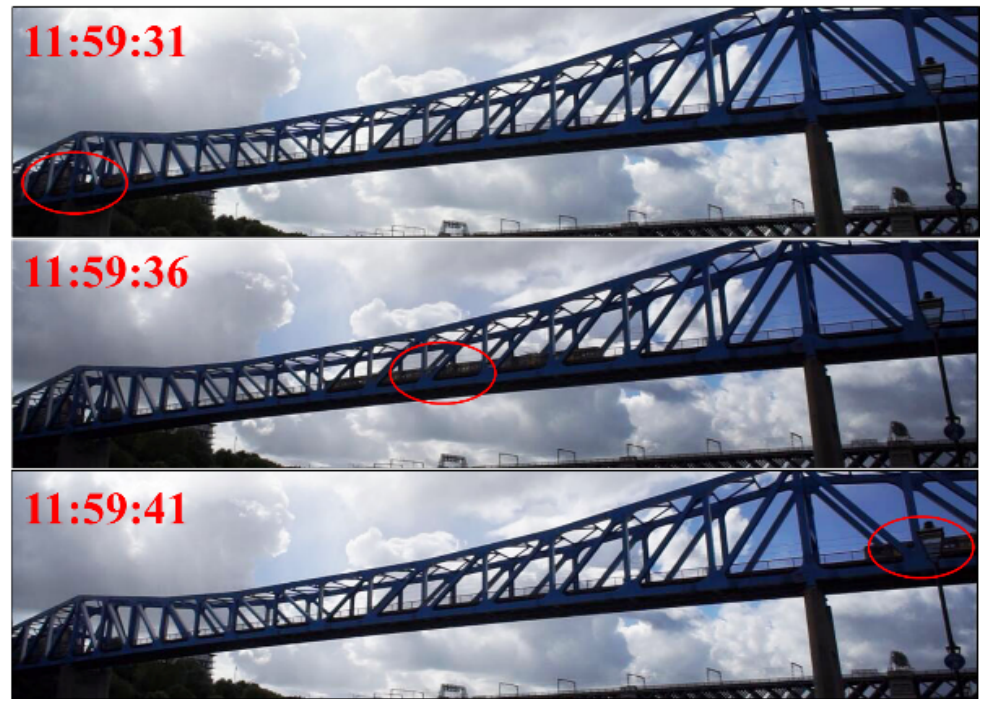

Figure 6. Three video frames showing the train crossing the bridge.

Negative displacement values mean targets become closer to the radar system along the LOS direction relative to their reference positions. In this case, negative deformation implies a lowering of the bridge 
superstructure and positive deformation means uplift, as the radar was tilted up to observe the bridge. The first obvious deformation signal appears in "115833-115933" and shows that the middle bridge superstructure lowers by approximately $2 \mathrm{~mm}$ and the near portion of the bridge superstructure uplifting by several submillimetres, suggesting the train was primarily located at the far side of the bridge during the period of the acquisition "115933". The next deformation map "115833-115943", shows an uplift of approximately 1.4 to $0.5 \mathrm{~mm}$ for the superstructure from ranges between 95 to $150 \mathrm{~m}$, respectively, and subsidence occurring at the very nearside, which implies the train was primarily located at the near side of the bridge during the period of the acquisition "115943". The deformation signals are therefore consistent with the synchronous video recording.

Note that the aliasing of the near two piers with the nearside bridge superstructure exists due to the inherent azimuth ambiguities caused by the oblique view geometry of the radar system. It is possible that results are degraded with respect to the corresponding area as a consequence. Moreover, the bridge might have vibrated with a certain magnitude whilst the train crossed. In SAR mode, it is not possible to measure higherfrequency vibrations of the bridge, however, the experiment demonstrates the potential of GBSAR time series analysis for monitoring sites with a fast-changing rate.

\section{Analysis and discussions}

\subsection{Analysis coherent pixels selection}

The proposed approach was compared with: (a) the selection of coherent pixels using a single pair of GBSAR images; (b) the PS selection based on ADI using a stack of GBSAR SLC images.

Based on the same coherence estimation algorithm and threshold value used in the time series analysis, the coherence between the first and the last SLCs in Dataset I was calculated. Coherent pixels were then selected on the basis of the single pair of GBSAR images, as shown in Figure 7. By comparing the coherence of the single pair of GBSAR images (Figure 7(a)) with the mean coherence image of the entire network of interferograms formed in the time series analysis (Figure 7(b)), it is clear that the interferogram suffers temporal decorrelations. The number of coherent pixels selected in this single interferogram was 2,340 (Figure 7 (c)), which is much fewer than that $(5,068$, Figure 3(a)) achieved by the proposed selection method. The displacement map produced by single-pair interferometry is shown in Figure 7(d). With the exception of several pixels near the top-left position $(-110,385)$ that show approximately $2.5 \mathrm{~mm}$ of displacement and is probably due to residual atmospheric artefacts, no other significant signals can be observed. However, the limited density of coherent pixels can result in difficulties in phase unwrapping due to local under-sampling and phase discontinuities [36, 37], and thus impede the interpretation of the GBSAR data. 


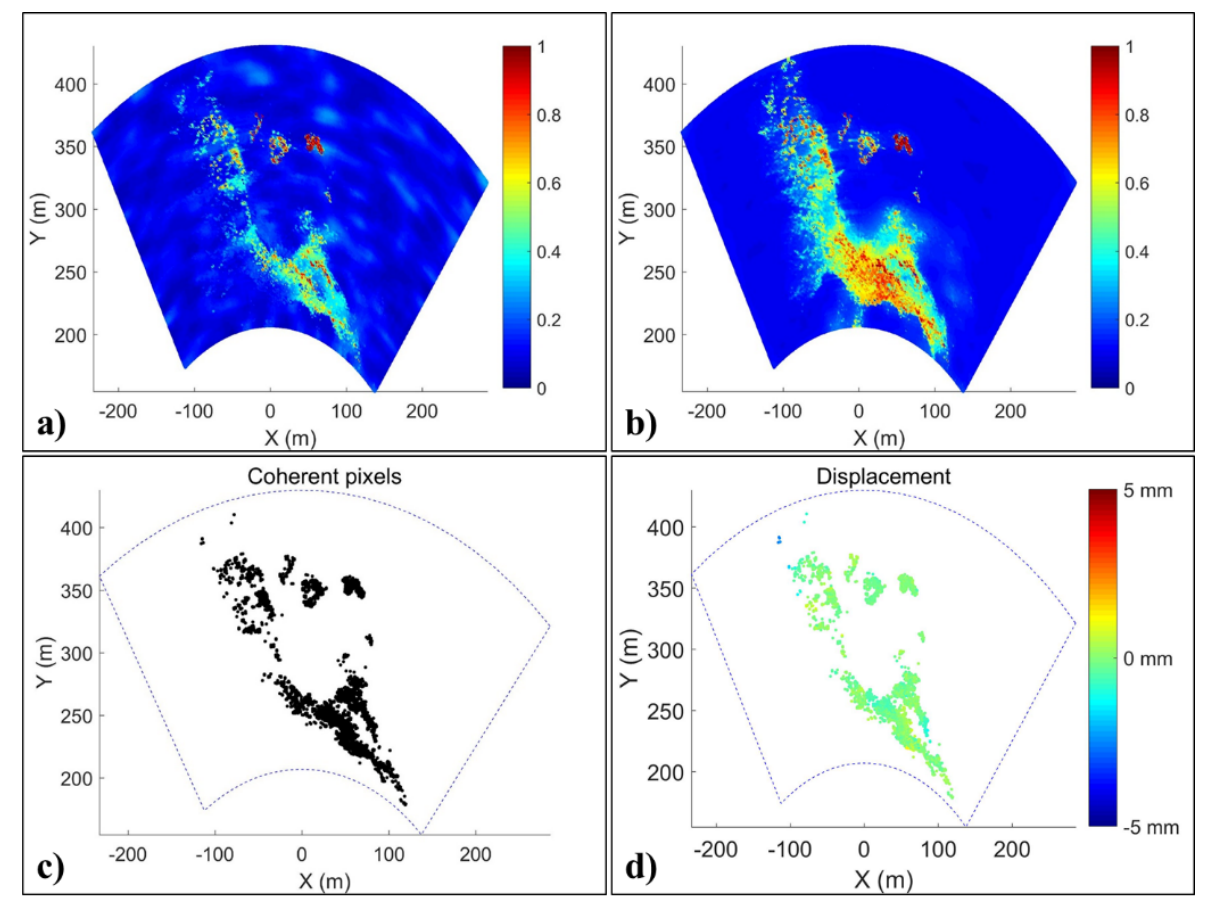

Figure 7. (a) Coherence between the first and the last SLCs in Dataset I. (b) The mean coherence of the entire interferogram network, shown for comparison. (c) Coherent pixels selected based on the single-pair of GBSAR images. (d) Displacement over the two-hour observation period, achieved by single-pair interferometry.

For Dataset II, the analysis focused on the two key images ("115933" and "115943") acquired when the train was on the bridge. The selection of coherent pixels and the inversion of deformation were achieved by using this single pair of acquisitions and the same parameters used in the time series analysis. Figure 8 shows the coherence and displacement maps between the two acquisitions. It can be observed that the decorrelation effect for this 10-second data is limited and the results achieved by the single-pair interferometry and the time series analysis are generally consistent, although over $20 \%$ coherent pixels are obtained using the time series analysis approach.
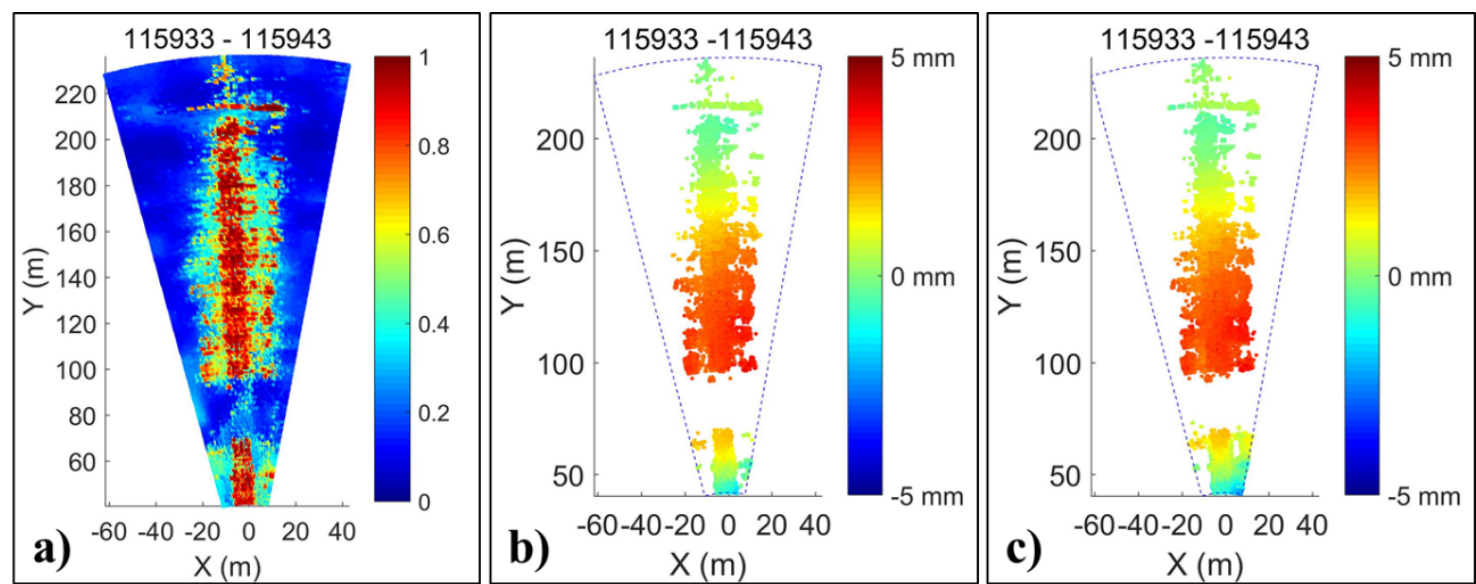

Figure 8. Results of the pair of acquisitions: "115933" and "115943". (a) The coherence map. (b) The displacement map derived with the single-pair interferometry (number of coherent pixels: 4,742). (c) The displacement map derived with the proposed times series analysis (number of coherent pixels: 5,720), namely the difference between the $6^{\text {th }}$ and $7^{\text {th }}$ maps in Figure 5.

PS detection was also conducted using Dataset I, as depicted in Figure 9. A pixel with an ADI lower than 0.25 (a commonly used threshold) is considered as a PS target. 1,312 PSs were detected from Dataset I. By 
comparing the detected PSs in Figure 9(b) and coherent pixels in Figure 3(a), the former is found to be only a subset of the latter, which demonstrates the feasibility of the proposed method in selecting persistent scatterers. In addition, the ADI threshold values were also increased to investigate the performance of PS detection. Figure 9(c) shows that several PSs were detected from shadow zones and sea areas where the ADI threshold was set as 0.30. The situation worsened (Figure 9(d)) when the ADI threshold was set as 0.35 . This result demonstrates an advantage of the proposed approach on selecting dense and reliable coherent pixels over the ADI-based PS selection technique, which was also confirmed by the PS analysis on Dataset II, as depicted in Figure 10.

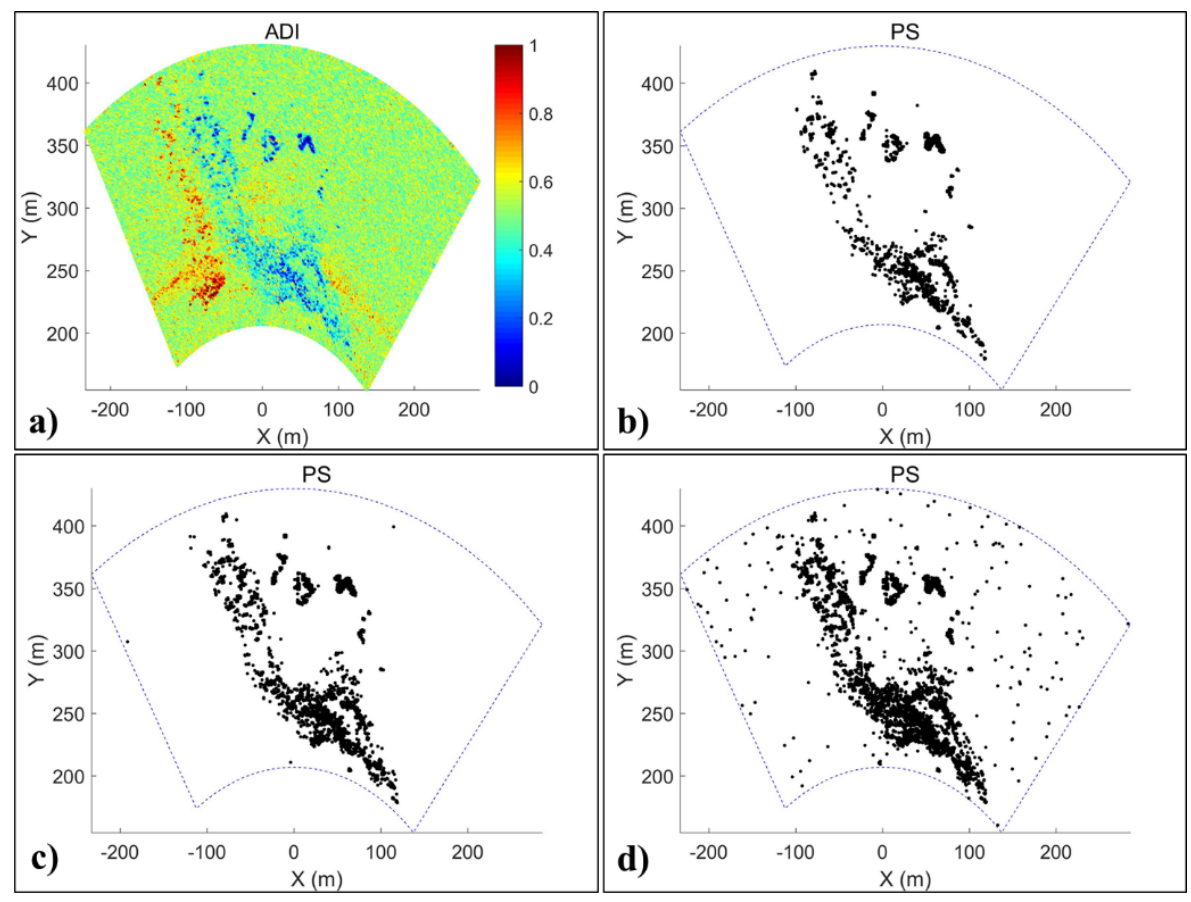

Figure 9. Dataset I: PS detection via ADI. (a) ADI for Dataset I. (b) PSs with ADI $<0.25$ (number of PS points: 1,312). (c) PSs with $\mathrm{ADI}<0.30$ (number of PS points: 2,052). (d) PSs with $\mathrm{ADI}<0.35$ (number of PS points: $3,273)$.
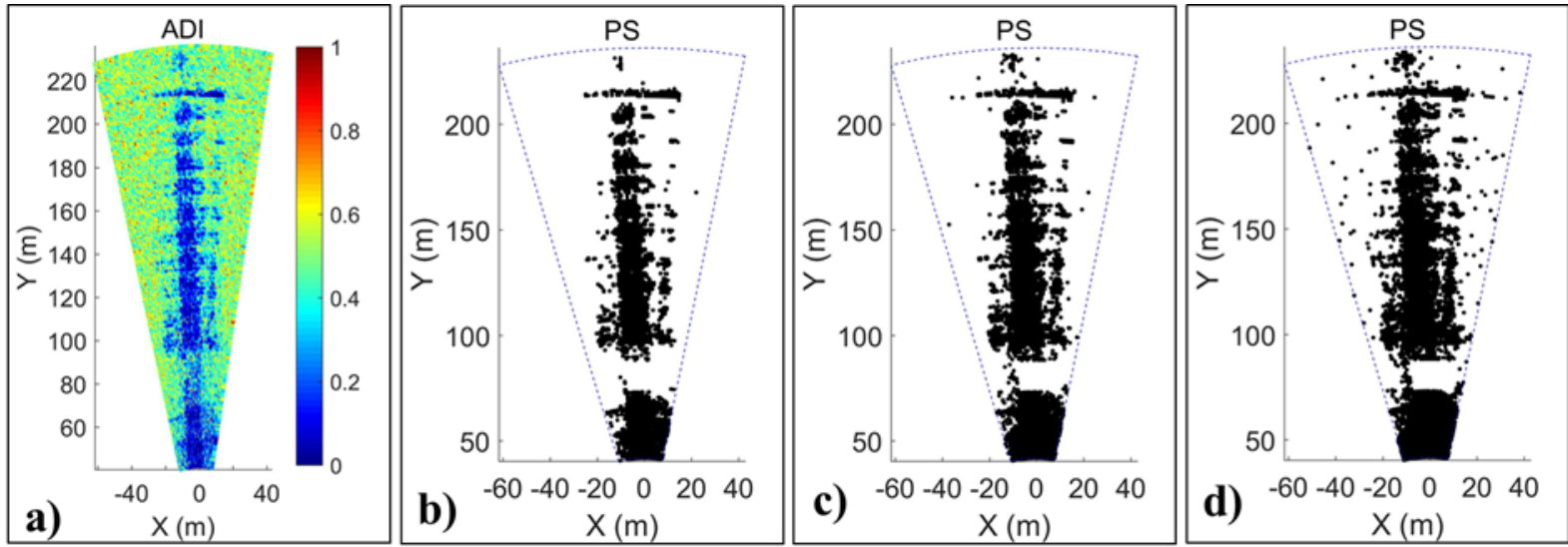

Figure 10. Dataset II: PS detection via ADI. (a) ADI for Dataset II. (b) PSs with $A D I<0.20$ (number of PS points: 3,924). (c) PSs with $\mathrm{ADI}<0.25$ (number of PS points: 5,016). (d) PSs with $\mathrm{ADI}<0.30$ (number of PS points: 6,135$)$.

\subsection{Justification of relevant parameters}

There are two main parameters in the proposed method for the selection of coherent pixels: (a) the 
coherence threshold; (b) the temporal baseline constraint. These two parameters are actually quite straightforward. A higher coherence means better statistics for the interferometric phase. Accordingly, a higher coherence threshold will lead to fewer pixels with better interferometric phase quality. As mentioned previously, the density of coherent pixels plays an important role in the time series analysis, and a tradeoff between the quantity and quality of coherent pixels should be exercised in practice.

In addition, the temporal baseline directly determines the network of interferograms, thus it is a key factor in GBSAR time series analysis for deformation monitoring. Therefore, the entire GBSAR time series analysis based on Datasets I and II with different temporal baseline constraints was performed to investigate the effects of this parameter on the number of selected coherent pixels, the RMS of inversion precision and the computational efficiency. The results are illustrated in Figure 11.

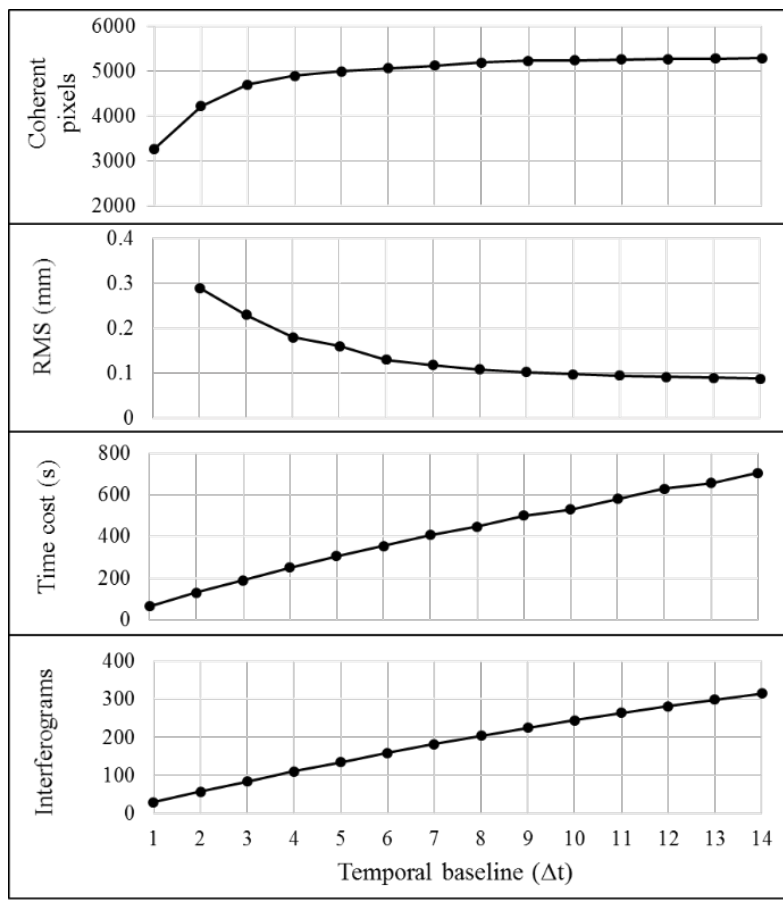

(a)

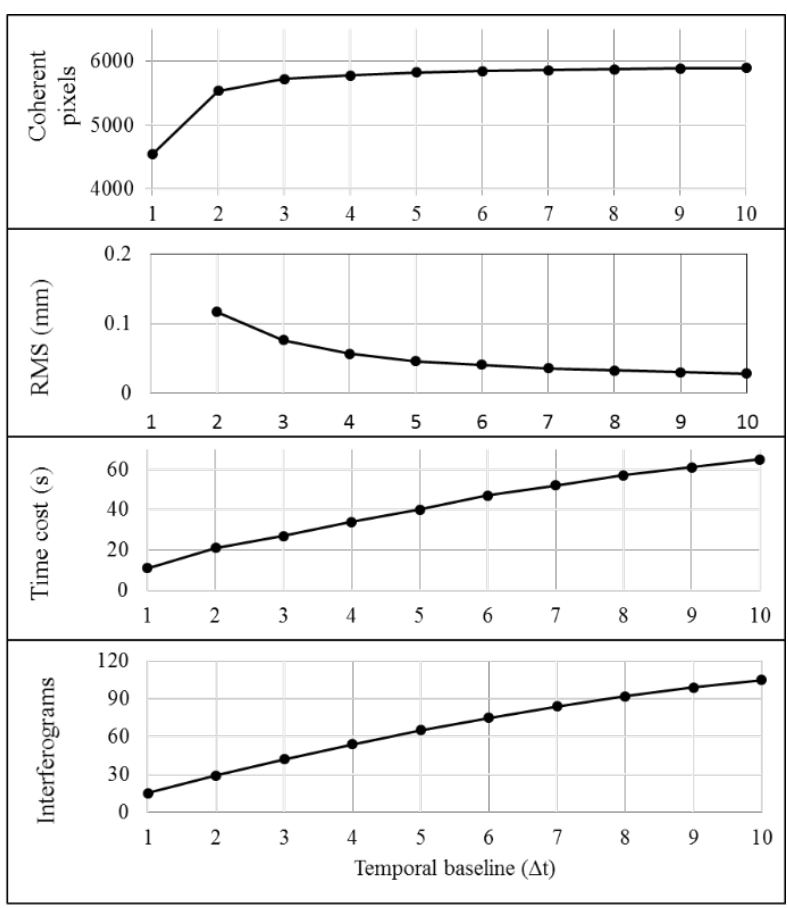

(b)

Figure 11. Influence of the temporal baseline on the selection of coherent pixels, inversion precision and computational efficiency. $\Delta \mathrm{t}$ is the repeat interval (4 minutes for Dataset I and 10 seconds for Dataset II). The RMS of inversion precision is not applicable when the temporal baseline is set as $1 \Delta t$ as a zero-redundancy network is formed in this case. (a) Dataset I. (b) Dataset II.

Unsurprisingly, the number of coherent pixels increases with the temporal baseline. This is because a longer temporal baseline leads to more redundancy in the network and thus offers more coherent opportunities for a pixel to construct a connected network and achieve a full-rank matrix B. It is worth noting, however, that the improvement achieved by increasing the temporal baseline is not significant after a certain value is reached since the majority of coherent pixels have been excavated and temporal decorrelation also becomes significant.

Moreover, a longer temporal baseline will lead to more redundancy in the network and obtain more reliable results, but at the expense of the computational efficiency. Similarly, the improvement in the achieved precision is limited when the temporal baseline reaches a certain value (around 7 for Dataset I and 5 for Dataset II). However, processing time keeps increasing with the temporal baseline, and is approximately proportional to the number of interferograms being processed. Moreover, there is little purpose to enlarging the temporal baseline once the density of coherent pixels reaches a density sufficient for the interpretation requirements. Therefore, in practice, the selection of the temporal baseline is made on the basis of computational efficiency 
and the required accuracy.

\section{Conclusions}

This study has proposed a criterion for the selection of coherent pixels from a redundant network of interferograms. The proposed approach has been integrated into an entire pipeline for GBSAR time series analysis in deformation monitoring. The following conclusions are reached from the experiments reported in this paper:

(1) Experiments were performed on two continuous observational datasets, one of a coastal cliff and the other for a rail bridge. The cliff was stable during the monitoring period while deformation signals of several $\mathrm{mm}$ were detected when a train crossed the bridge. The time series estimation for both datasets reached up to a few sub-millimeters, which supports the feasibility of the proposed method for GBSAR deformation monitoring purpose.

(2) Temporal decorrelation is a serious issue in SAR interferometric measurement [5], especially for shortwavelength GBSAR systems. In comparison with the single-master InSAR technique, the proposed approach is able to largely overcome the temporal decorrelation problem and ensure a successful interpretation.

(3) The proposed approach enables the selection of not only qualified partially coherent pixels, but also all persistent scatterers. The proposed method makes the most of redundant observations and allows an adjustment to obtain a final reliable value for the unknown. Finally, a reliable solution was achieved in least squares inversion.

On the basis of these findings, the proposed approach appears to facilitate a range of deformation monitoring applications to which GBSAR is suited. Although the experimental datasets were acquired continuously under zero-base line mode, the proposed selection criterion is potentially suitable for any well co-registered discontinuous SAR datasets.

\section{Acknowledgements}

This research was supported by a China Scholarship Council (CSC) studentship (No. 201506270153) held by Zheng Wang at Newcastle University, UK. The work was also supported by the National Environment Research Council (NERC) through the Centre for the Observation and Modelling of Earthquakes, Volcanoes and Tectonics (COMET, ref.: come30001), the LiCS project (ref. NE/K010794/1), the ESA-MOST DRAGON-4 project (ref. 32244), and the Hunan Province Key Laboratory of Coal Resources Clean-Utilization and Mine Environment Protection, Hunan University of Science and Technology (Ref.: E21608 and E21802).

\section{Appendix A: Inversion precision}

The proposed approach ensures that each pixel is associated with a full-rank coefficient matrix $\mathbf{B}$. With redundant interferometric phase, the optimal estimation of the incremental time series of phase change (i.e., $\widehat{\boldsymbol{\Phi}})$ for each pixel can be performed based on Equation (6) via least squares. The phase residuals in the least squares inversion are:

$$
\mathbf{V}_{L \times 1}=\mathbf{B}_{L \times N} \widehat{\boldsymbol{\Phi}}_{N \times 1}-\boldsymbol{\delta} \boldsymbol{\Phi}_{L \times 1} .
$$

The root mean square of phase residuals for this pixel is:

$$
\sigma_{0}=\sqrt{\frac{\mathbf{v}^{\mathrm{T}} \mathbf{V}}{r}}=\sqrt{\frac{\mathbf{v}^{\mathrm{T}} \mathbf{V}}{n-N+1}}
$$

where $r$ is the number of redundancies and $n$ is the number of coherence occurrences in the redundant network. 
Accordingly, the covariance matrix of the estimated $\widehat{\boldsymbol{\Phi}}_{N \times 1}$ can be calculated by:

$$
\mathrm{D}_{\widehat{\Phi} \widehat{\Phi}}=\sigma_{0} \sqrt{\left(\mathbf{B}^{\mathrm{T}} \mathbf{B}\right)^{-1}} \text {. }
$$

As the final cumulative displacement is obtained by removing atmospheric variations from the sum of them, an estimator $\hat{d}$ is introducedwhich is the sum of the cumulative displacement and atmospheric variation between the first and the last acquisition:

$$
\hat{d}=-\frac{\lambda}{4 \pi} \sum_{k=0}^{N-1}\left(\hat{\varphi}_{t_{k} t_{k+1}}^{d i s}+\hat{\varphi}_{t_{k} t_{k+1}}^{a t m}\right)=\mathbf{F}_{1 \times N} \widehat{\boldsymbol{\Phi}}_{N \times 1},
$$

where $\mathbf{F}=\left[\begin{array}{lll}-\lambda / 4 \pi & \cdots & -\lambda / 4 \pi\end{array}\right]$. The theoretical precision of $\widehat{\mathrm{d}}$ is used as the precision indicator in the least squares estimation for each pixel, which is calculated by:

$$
\sigma_{\hat{d} \hat{d}}=\sqrt{\mathrm{D}_{\hat{d} \hat{d}}}=\sqrt{\mathbf{F D}_{\widehat{\mathbf{\Phi}} \hat{\mathbf{\Phi}}} \mathbf{F}^{\mathrm{T}}} .
$$

\section{Reference}

[1] M. Crosetto, O. Monserrat, G. Luzi, N. Devanthéry, M. Cuevas-González, and A. Barra, "DATA PROCESSING AND ANALYSIS TOOLS BASED ON GROUND-BASED SYNTHETIC APERTURE RADAR IMAGERY," ISPRS-International Archives of the Photogrammetry, Remote Sensing and Spatial Information Sciences, pp. 593-596, 2017.

[2] M. Crosetto, O. Monserrat, G. Luzi, M. Cuevas, and N. Devanthéry, "Deformation Monitoring Using GroundBased SAR Data," in Engineering Geology for Society and Territory-Volume 5: Springer, 2015, pp. 137-140.

[3] O. Monserrat, M. Crosetto, and G. Luzi, "A review of ground-based SAR interferometry for deformation measurement," ISPRS Journal of Photogrammetry and Remote Sensing, vol. 93, pp. 40-48, 2014.

[4] D. Wujanz, F. Neitzel, H. Hebel, J. Linke, and W. Busch, "Terrestrial radar and laser scanning for deformation monitoring: first steps towards assisted radar scanning," ISPRS Annals of Photogrammetry, Remote Sensing and Spatial Information Sciences, vol. 2, no. 5/W2, pp. 325-330, 2013.

[5] R. Caduff, F. Schlunegger, A. Kos, and A. Wiesmann, "A review of terrestrial radar interferometry for measuring surface change in the geosciences," Earth Surface Processes and Landforms, vol. 40, no. 2, pp. 208-228, 2015.

[6] A. Ferretti, C. Prati, and F. Rocca, "Permanent scatterers in SAR interferometry," Geoscience and Remote Sensing, IEEE Transactions on, vol. 39, no. 1, pp. 8-20, 2001.

[7] R. Lanari et al., "An overview of the small baseline subset algorithm: A DInSAR technique for surface deformation analysis," Pure and Applied Geophysics, vol. 164, no. 4, pp. 637-661, 2007.

[8] A. Hooper, D. Bekaert, K. Spaans, and M. Arıkan, "Recent advances in SAR interferometry time series analysis for measuring crustal deformation," Tectonophysics, vol. 514, pp. 1-13, 2012.

[9] P. Blanco-Sanchez, J. J. Mallorquí, S. Duque, and D. Monells, "The coherent pixels technique (CPT): An advanced DInSAR technique for nonlinear deformation monitoring," Pure and Applied Geophysics, vol. 165, no. 6, pp. 1167-1193, 2008.

[10] R. Iglesias, J. J. Mallorqui, and P. López-Dekker, "DInSAR pixel selection based on sublook spectral correlation along time," Geoscience and Remote Sensing, IEEE Transactions on, vol. 52, no. 7, pp. 3788-3799, 2014.

[11] R. Bamler and P. Hartl, "Synthetic aperture radar interferometry," Inverse problems, vol. 14, no. 4, p. R1, 1998.

[12] B. Osmanoğlu, F. Sunar, S. Wdowinski, and E. Cabral-Cano, "Time series analysis of InSAR data: Methods and trends," ISPRS Journal of Photogrammetry and Remote Sensing, vol. 115, pp. 90-102, 2016.

[13] P. Berardino, G. Fornaro, R. Lanari, and E. Sansosti, "A new algorithm for surface deformation monitoring based on small baseline differential SAR interferograms," IEEE Transactions on Geoscience and Remote Sensing, vol. 40, no. 11, pp. 2375-2383, 2002.

[14] R. Lanari, O. Mora, M. Manunta, J. J. Mallorquí, P. Berardino, and E. Sansosti, "A small-baseline approach for 
investigating deformations on full-resolution differential SAR interferograms," IEEE Transactions on Geoscience and Remote Sensing, vol. 42, no. 7, pp. 1377-1386, 2004.

[15] D. Perissin and T. Wang, "Repeat-pass SAR interferometry with partially coherent targets," IEEE Transactions on Geoscience and Remote Sensing, vol. 50, no. 1, pp. 271-280, 2012.

[16] P. Shanker, F. Casu, H. A. Zebker, and R. Lanari, "Comparison of persistent scatterers and small baseline timeseries InSAR results: a case study of the San Francisco Bay Area," IEEE Geoscience and Remote Sensing Letters, vol. 8, no. 4, pp. 592-596, 2011.

[17] K. Spaans and A. Hooper, "InSAR processing for volcano monitoring and other near - real time applications," Journal of Geophysical Research: Solid Earth, vol. 121, no. 4, pp. 2947-2960, 2016.

[18] M. Crosetto, E. Biescas, J. Duro, J. Closa, and A. Arnaud, "Generation of advanced ERS and Envisat interferometric SAR products using the stable point network technique," Photogrammetric Engineering \& Remote Sensing, vol. 74, no. 4, pp. 443-450, 2008.

[19] C. Prati, A. Ferretti, and D. Perissin, "Recent advances on surface ground deformation measurement by means of repeated space-borne SAR observations," Journal of Geodynamics, vol. 49, no. 3, pp. 161-170, 2010.

[20] H. A. Zebker and Y. Lu, "Phase unwrapping algorithms for radar interferometry: residue-cut, least-squares, and synthesis algorithms," JOSA A, vol. 15, no. 3, pp. 586-598, 1998.

[21] K. Takahashi, M. Matsumoto, and M. Sato, "Continuous observation of natural-disaster-affected areas using ground-based SAR interferometry," Selected Topics in Applied Earth Observations and Remote Sensing, IEEE Journal of, vol. 6, no. 3, pp. 1286-1294, 2013.

[22] M. Crosetto, O. Monserrat, G. Luzi, M. Cuevas-González, and N. Devanthéry, "Discontinuous GBSAR deformation monitoring," ISPRS Journal of Photogrammetry and Remote Sensing, vol. 93, pp. 136-141, 2014.

[23] Z. Li, E. J. Fielding, and P. Cross, "Integration of InSAR time-series analysis and water-vapor correction for mapping postseismic motion after the 2003 Bam (Iran) earthquake," Geoscience and Remote Sensing, IEEE Transactions on, vol. 47, no. 9, pp. 3220-3230, 2009.

[24] L. Iannini and A. M. Guarnieri, "Atmospheric phase screen in ground-based radar: Statistics and compensation," IEEE Geoscience and Remote Sensing Letters, vol. 8, no. 3, pp. 537-541, 2011.

[25] R. Touzi, A. Lopes, J. Bruniquel, and P. W. Vachon, "Coherence estimation for SAR imagery," IEEE Transactions on Geoscience and Remote Sensing, vol. 37, no. 1, pp. 135-149, 1999.

[26] C.-A. Deledalle, L. Denis, and F. Tupin, "Nl-insar: Nonlocal interferogram estimation," IEEE Transactions on Geoscience and Remote Sensing, vol. 49, no. 4, pp. 1441-1452, 2011.

[27] Z. Wang, Z. Li, and J. Mills, "A New Non-local Method for Ground-based SAR Deformation Monitoring," Manuscript submitted for publication, 2018.

[28] R. Abdelfattah and J.-M. Nicolas, "Interferometric SAR coherence magnitude estimation using second kind statistics," IEEE Transactions on Geoscience and Remote Sensing, vol. 44, no. 7, pp. 1942-1953, 2006.

[29] M. Jiang et al., "The Improvement for Baran Phase Filter Derived From Unbiased InSAR Coherence," Selected Topics in Applied Earth Observations and Remote Sensing, IEEE Journal of, vol. 7, no. 7, pp. 3002-3010, 2014.

[30] M. Costantini and P. A. Rosen, "A generalized phase unwrapping approach for sparse data," in Geoscience and Remote Sensing Symposium, 1999. IGARSS'99 Proceedings. IEEE 1999 International, 1999, vol. 1, pp. 267-269: IEEE.

[31] R. Iglesias et al., "Atmospheric phase screen compensation in ground-based SAR with a multiple-regression model over mountainous regions," Geoscience and Remote Sensing, IEEE Transactions on, vol. 52, no. 5, pp. 2436-2449, 2014.

[32] L. Pipia, X. Fabregas, A. Aguasca, and C. Lopez-Martinez, "Atmospheric artifact compensation in ground-based DInSAR applications," IEEE Geoscience and Remote Sensing Letters, vol. 5, no. 1, pp. 88-92, 2008.

[33] S. Rödelsperger and A. Meta, "MetaSensing's FastGBSAR: ground based radar for deformation monitoring," in 
SAR Image Analysis, Modeling, and Techniques XIV, 2014, vol. 9243, p. 924318: International Society for Optics and Photonics.

[34] S. Rödelsperger, Real-time processing of ground based synthetic aperture radar (GB-SAR) measurements (no. 33). Technische Universität Darmstadt, Fachbereich Bauingenieurwesen und Geodäsie, 2011.

[35] R. F. Hanssen, Radar interferometry: data interpretation and error analysis. Springer Science \& Business Media, 2001.

[36] A. Hooper and H. A. Zebker, "Phase unwrapping in three dimensions with application to InSAR time series," JOSA A, vol. 24, no. 9, pp. 2737-2747, 2007.

[37] Z. Perski, R. Hanssen, A. Wojcik, and T. Wojciechowski, "InSAR analyses of terrain deformation near the Wieliczka Salt Mine, Poland," Engineering Geology, vol. 106, no. 1-2, pp. 58-67, 2009. 